\title{
Malignant Progression of Diffuse Low-grade Gliomas: A Systematic Review and Meta-analysis on Incidence and Related Factors
}

\author{
Satoshi NAKASU ${ }^{1,2}$ and Yoko NAKASU ${ }^{2,3}$ \\ ${ }^{1}$ Division of Neurosurgery, Omi Medical Center, Kusatsu, Japan \\ ${ }^{2}$ Department of Neurosurgery, Shiga University of Medical Science, Ohtsu, Japan \\ ${ }^{3}$ Division of Neurosurgery, Shizuoka Cancer Center, Nagaizumi, Japan
}

\begin{abstract}
Malignant progression of diffuse low-grade glioma (LGG) is a critical event affecting patient survival; however, the incidence and related factors have been inconsistent in literature. According to the PRISMA guidelines, we systematically reviewed articles from 2009, meta-analyzed the incidence of malignant progression, and clarified factors related to the transformation. Forty-one articles were included in this study ( $n=7,122$; $n$, number of patients). We identified two definitions of malignant progression: histologically proven (Htrans) and clinically defined (Ctrans). The malignant progression rate curves of Htrans and Ctrans were almost in parallel when constructed from the results of metaregression by the mean follow-up time. The true transformation rate was supposed to lie between the two curves, approximately $40 \%$ at the 10 -year mean follow-up. Risk of malignant progression was evaluated using hazard ratio (HR). Pooled HRs were significantly higher in tumors with a larger preand postoperative tumor volume, lower degree of resection, and notable preoperative contrast enhancement on magnetic resonance imaging than in others. Oligodendroglial histology and IDH mutation (IDHm) with 1p/19q codeletion (Codel) also significantly reduced the HRs. Using Kaplan-Meier curves from eight studies with molecular data, we extracted data and calculated the 10-year malignant progression-free survival (10yMPFS). The 10yMPFS in patients with IDHm without Codel was $30.4 \%$ (95\% confidence interval [95\% CI]: 22.2-39.0) in Htrans and 38.3\% (95\% CI: 32.3-44.3) in Ctrans, and that with IDHm with Codel was 71.7\% (95\% CI: 61.7-79.5) in Htrans and 62.5\% (95\% CI: 55.9-68.5) in Ctrans. The effect of adjuvant radiotherapy or chemotherapy could not be determined.
\end{abstract}

Keywords: low-grade glioma, diffuse astrocytoma, oligodendroglioma, malignant transformation

\section{Introduction}

The incidence rate of diffuse low-grade gliomas (LGGs) is estimated to be approximately 0.8 cases per 100,000 population. ${ }^{1)}$ LGGs histologically consist of diffuse astrocytomas and oligodendrogliomas. However, the histomorphological diagnosis of astrocytomas, oligodendrogliomas, and mixed gliomas has not been always distinct. The recent advent of molecular diagnoses, including IDH mutation (IDHm) and $1 \mathrm{p} / 19 \mathrm{q}$ codeletion (Codel), has resulted in the development of a clearer classification system of LGGs. The 2016 WHO classification employed molecular markers in the diagnosis of LGG." Although IDH wild-type (IDHw)
LGG may be a heterogeneous group, the new classification (2021) defines IDHw diffuse glioma with specific molecular features as glioblastoma. ${ }^{2}$

LGGs are slowly growing tumors and often lack symptoms, except for seizures. The frequency of incidental cases is increasing with the spread of imaging studies, despite the low prevalence of incidental LGGs $(0.064 \%$ of imaging studies)." Although the "wait and see" approach was often selected historically, long-term follow-up has revealed that the majority of these lesions cause malignant transformation (malignant progression from low-grade malignancy), which results in death. ${ }^{4.5)}$ Some of the researchers advocated an early diagnosis and a preventive surgical

Received September 24, 2021; Accepted December 13, 2021

Copyright (C) 2022 The Japan Neurosurgical Society

This work is licensed under a Creative Commons Attribution-NonCommercial-NoDerivatives International License. 

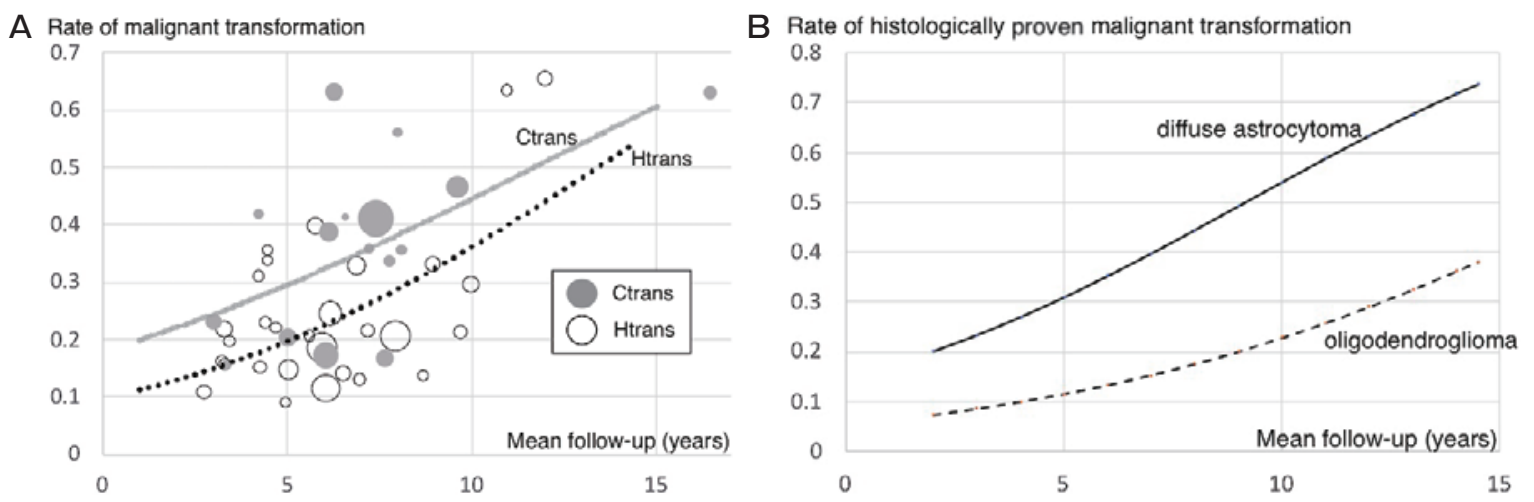

Fig. 1 A. Almost parallel regression lines of malignant transformation in Ctrans and Htrans. Size of circle = number of cases. B. The rate of malignant transformation by Htrans in diffuse astrocytomas is higher than in oligodendrogliomas. Ctrans, clinically defined malignant transformation; Htrans, histologically proved malignant transformation. (Curves are made from back transformation of logit transformed values in Table 1.)

treatment to improve the outcomes of LGGs, suggesting the relevance of a tailored screening policy. ${ }^{6}$ Early maximal safe resection while preserving the eloquent brain areas is currently considered to be a better treatment option.

Patients with malignant transformed glioma exhibited a much worse overall survival (OS) than those who still had low-grade histology at recurrence. ${ }^{8)}$ The median OS in patients with malignant transformed glioma was reported to be approximately 2 years after transformation. ${ }^{9-11)}$ Malignant transformation of diffuse LGG is a critical event influencing the patient survival, but the incidence and timecourse are inconsistently reported in the literature with the 10-year malignant progression-free survival (MPFS) rates ranging from $22.4 \%^{12}$ to $60.6 \%^{5)}$. Although the cause of this variation is unclear, previous studies often lacked molecular data and demonstrated heterogeneity in a rate of malignant transformation (MaligR) due to differences among studies in the criteria for malignant transformation, histological diagnosis, and treatment strategies.

We reviewed articles systematically and conducted their meta-analysis.

\section{Materials and Methods}

\section{Literature search and data extraction}

A literature search was conducted independently by two of the authors. The PRISMA search flow diagram is outlined in Supplementary Figure 1. We searched for relevant English articles using the keywords "low-grade glioma," "astrocytoma" or "oligodendroglioma" and "malignant or anaplastic transformation” in PubMed, Scoups, and Google Scholar and published in 2009 or later, when the first article on IDH mutation in gliomas was published. Studies including $\geq 50$ LGG cases were selected, as were those with $\geq$ 40 diffuse astrocytomas or oligodendrogliomas. Pediatric cases, spinal tumors, and studies limited to a specific location were excluded. The exact search strategy is described in Supplementary Figure 1. The search was performed on
April 24, 2021. Two articles were added after a manual search on August 5, 2021.

From each study, we collected data on the age, sex, mean follow-up time (MFTime), the number of subjects, and malignant transformations. When only the median follow-up data were available, the mean was calculated using the following equation obtained from eight studies that described both the mean and median values $\left(\mathrm{R}^{2}=0.99\right)$ : mean $=1.14 \times$ median. The astrocytoma rate (AstroR) and pure oligodendroglioma rate (OligR) were calculated as total numbers of diffuse astrocytomas or oligodendrogliomas, respectively, divided by the total number of cases; mixed gliomas were not included. Treatment-related factors, including the extent of removal, and number of adjuvant radiotherapy and chemotherapy sessions were also recorded. The rate of gross total removal (GTRate) was calculated, defined as resection of $\geq 90 \%$ of the volume. Data on molecular tests, results of IDHm, and Codel were extracted whenever available.

We found two definitions of malignant transformation described in the literature. One was histologically proven (Htrans), which exhibited anaplastic transformation compatible with higher-grade glioma, and the other was clinically defined (Ctrans). Ctrans included cases that showed new progressive contrast enhancement on magnetic resonance imaging (MRI) in addition to histologically confirmed cases.

We used hazard ratio (HR) to determine the effect of malignant transformation in the meta-analysis. The HRs for age, sex, Karnofsky performance status score (KPS), pre- and postoperative volume, extent of resection, adjuvant radiotherapy and chemotherapy, and molecular markers were prospectively collected. The HRs for contrast enhancement on MRI and eloquent location were added later.

Hazard ratios in univariate and multivariate analyses were separately extracted and meta-analyzed. In the retrieved articles, the age, KPS, and size of tumor were ana- 

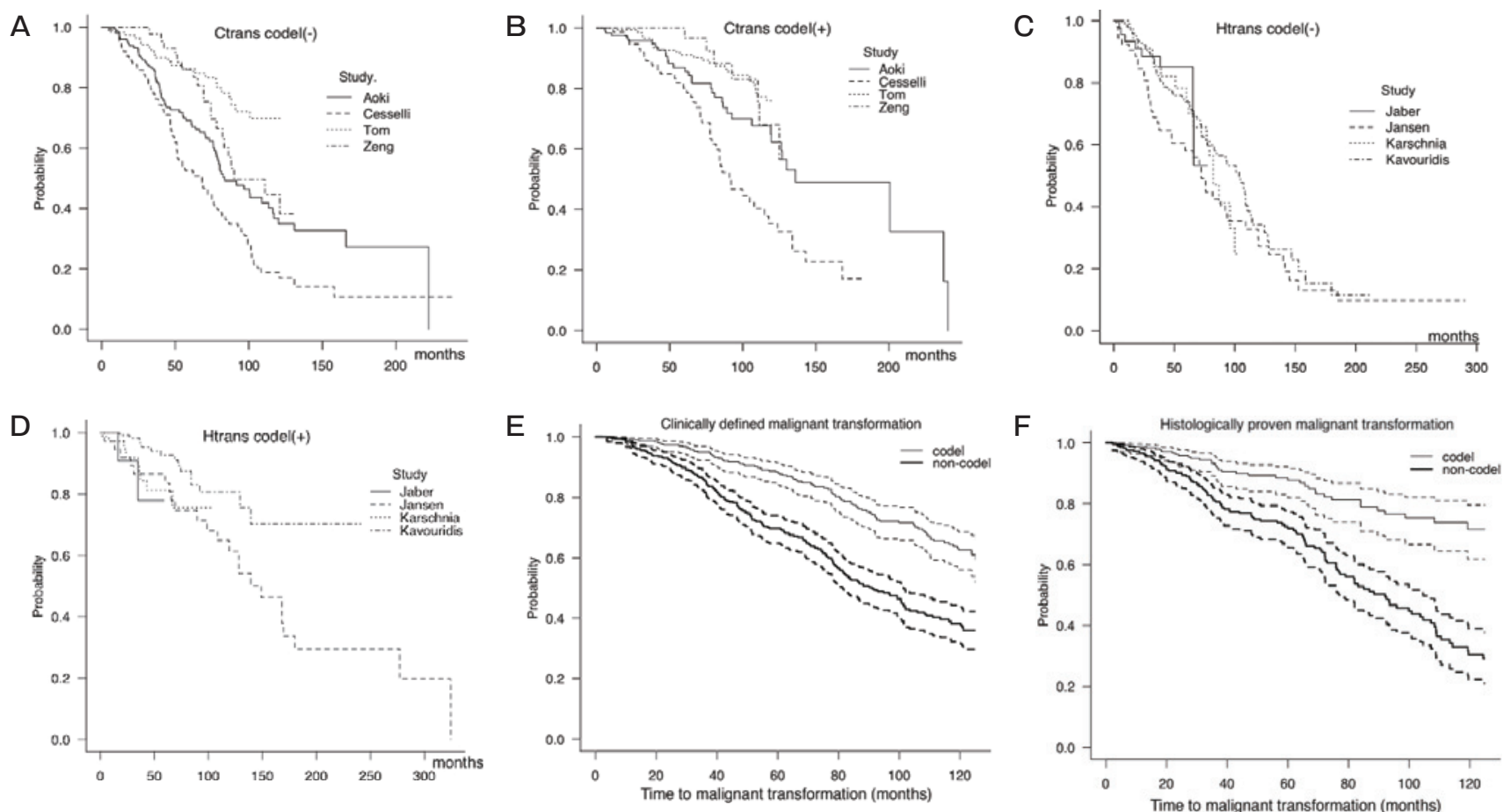

Fig. 2. Kaplan-Meier curves from extracted data. A. IDHm without Codel tumors in four studies with Ctrans. B. IDHm with Codel tumors in four studies with Ctrans. C. IDHm without Codel tumors in four studies with Htrans. D. IDHm with Codel tumors in four studies with Htrans. Synthesized Kaplan-Meier curves in Ctrans (E) and Htrans (F). Thick line, non-Codel tumors; thin line, Codel tumors; dotted line, $95 \%$ confidence interval.

IDHm, IDH mutant; Codel, 1p/19q codeletion; Ctrans, clinically defined malignant transformation; Htrans, histologically proved malignant transformation.

lyzed in different manners: categorized or continuous values (the approach most often used was adopted). When the HRs were not available, we calculated the missing values using log-rank tests or Kaplan-Meier curves according to the method of Tienarry et al. ${ }^{13)}$ We used WebPlotDigitizer (https://automeris.io/WebPlotDigitizer/) to extract data from Kaplan-Meier curves. In one study, we compared the data extracted from the Kaplan-Meier curve with those provided in the supplementary table $1 .{ }^{14)}$ Curves obtained from the extracted data and actual data were very similar (Supplementary Figure 2). The calculated 10yMPFS in the extracted data was $62.1 \%$ (95\% confidence interval [95\% CI]: 48.3-73.3), whereas that in the actual data was $62.2 \%$ (95\% CI: 48.4-73.4) in the actual data.

This review did not involve direct studies on humans, so informed consent was not required.

\section{Risk of bias}

We used the JBI critical appraisal checklist for case series (https://jbi.global/critical-appraisal-tools). Publication bias was evaluated using a funnel plot when $\geq 10$ studies were incorporated. A linear regression analysis was used in the test for funnel plot asymmetry.

\section{Statistical analyses}

We used the packages of EZR, Meta, and Metafor in the R software program (v4.03) (https://www.r-project.org/) to perform the statistical analyses. A single-arm meta-analysis was employed to calculate percentages using a generalized linear mixed model. The results of HR for malignant transformation in each factor were combined using the inversevariance method with the random-effect method (DerSimonian and Laird method).

The reviewed studies were tested for heterogeneity $\left(I^{2}\right.$ statistic). Meta-regression analyses were performed to identify factors related to heterogeneity, typically combined with the mean follow-up time, as this duration was a strong factor influencing transformation. Analyses were performed using the rma.glmm function in the Meta software package. Two-sided $\mathrm{P}$ values $<0.05$ were considered to indicate statistical significance.

\section{Results}

We initially retrieved 144 articles after removing duplicates (Supplementary Figure 1). After a full-text assessment, we excluded articles that did not describe the total population size or number with malignant transformation or the follow-up period. Data from the same institute were 
Table 1 Summary of the data

\begin{tabular}{|c|c|c|}
\hline & Htrans & Ctrans \\
\hline Total population & 4261 (28 studies) * & 3714 (18 studies) * \\
\hline Males/females & 2384/1806 (27 studies) & 1971/1516 (17 studies) \\
\hline \multirow[t]{2}{*}{ Median age } & Mean $^{* *}: 41.9$ (9 studies) & Mean $^{* *}: 42.7$ (4 studies) \\
\hline & Median $^{* * * *}: 38.5$ (16 studies) & Median $^{* * * *}: 38.0$ (13 studies) \\
\hline Malignant transformation & 975 & 1320 \\
\hline Median mean follow-up (years) & $5.83[4.38-7.38]$ & $6.45[5.25-7.74]$ \\
\hline Median rate of total removal & $0.412[0.309-0.49]$ (25 studies) & $0.361[0.135-0.457]$ (17 studies) \\
\hline Median rate of adjuvant radiotherapy & $0.314[0.237-0.510]$ (25 studies) & $0.312[0.278-0.818]$ (17 studies) \\
\hline Median rate of adjuvant chemotherapy & $0.167[0.08-0.333]$ (22 studies) & $0.216[0.042-0.298]$ (16 studies) \\
\hline Median rate of astrocytoma & 0.493 [0.424-0.647] (28 studies) & $0.661[0.532-0.744]$ (16 studies) \\
\hline Median rate of oligodendroglioma & $0.353[0.241-0.456]$ (28 studies) & $0.320[0.184-0.421]$ (16 studies) \\
\hline Study number & $28^{*}$ & $18^{*}$ \\
\hline
\end{tabular}

Htrans, histologically proven malignant transformation; Ctrans, clinically defined malignant transformation: *, including five studies (912 patients) with information for both Htrans and Ctrans; **, median of the mean age values; ${ }^{* * *}$, median of the median age values.

Table 2 Results of a meta-regression analysis of malignant transformation

\begin{tabular}{|c|c|c|c|c|}
\hline \multicolumn{5}{|c|}{ Histologically proved transformation $(\mathrm{N}=28)$} \\
\hline & \multicolumn{4}{|l|}{ Meta-regression } \\
\hline Variables & Estimate & $95 \% \mathrm{CI}$ & Intercept & $95 \% \mathrm{CI}$ \\
\hline \multirow[t]{2}{*}{$\mathrm{m}$ follow-up } & $0.167, P=0.0003$ & 0.085 to 0.251 & -2.24 & -2.80 to -1.68 \\
\hline & \multicolumn{4}{|c|}{ Multi-variate meta-regression } \\
\hline Oligo-rate & $-0.991, P=0.018$ & -1.814 to -0.168 & -1.857 & -2.47 to -1.25 \\
\hline $\mathrm{m}$ follow-up & $0.163, \mathrm{P}<0.001$ & 0.087 to 0.240 & & \\
\hline Astro-rate & $1.341, \mathrm{P}=0.0014$ & 0.519 to 2.163 & -3.106 & -3.84 to -2.38 \\
\hline $\mathrm{m}$ follow-up & $0.193, \mathrm{P}<0.0001$ & 0.115 to 0.270 & & \\
\hline \multicolumn{5}{|c|}{ Clinically defined transformation $(\mathrm{N}=18)$} \\
\hline & \multicolumn{4}{|l|}{ Meta-regression } \\
\hline Variables & Estimate & $95 \% \mathrm{CI}$ & Intercept & $95 \% \mathrm{CI}$ \\
\hline $\mathrm{m}$ follow-up & $0.130, P=0.006$ & 0.0368 to 0.221 & -1.52 & -2.21 to -0.84 \\
\hline
\end{tabular}

excluded. However, we sometimes included studies from the same institute with a small amount of overlapping data or with data from different analyses. One extra study in 2008 was included because it was found to have more cases with necessary data than the searched article from the same institute. We ultimately selected 23 articles with data on Htrans, ${ }^{4,15-36)} 13$ with data on Ctrans, ${ }^{4,5,9,10,12,14,36-42)}$, and 5 with both. ${ }^{11,43-46)}$ One study included information from two institutes, ${ }^{40)}$ so we analyzed the data, separately (summarized data are in Table 1, and all data are in Supplemen- tary Table 1). Additional five studies were adopted only for analyses of the HR in forest plots. ${ }^{47-51)}$

\section{Risk of bias}

Studies were either retrospective case series or observational studies. The JBI tool comprises 10 items that evaluate the risk of bias (Supplementary Table 2). The studies were classified as having a low risk of bias when $\geq 8$ of the 10 questions were answered "yes," a moderate risk of bias when 6-7 of the 10 questions were answered "yes", and a 
Table 3 Pooled hazard ratios in factors related to malignant transformation

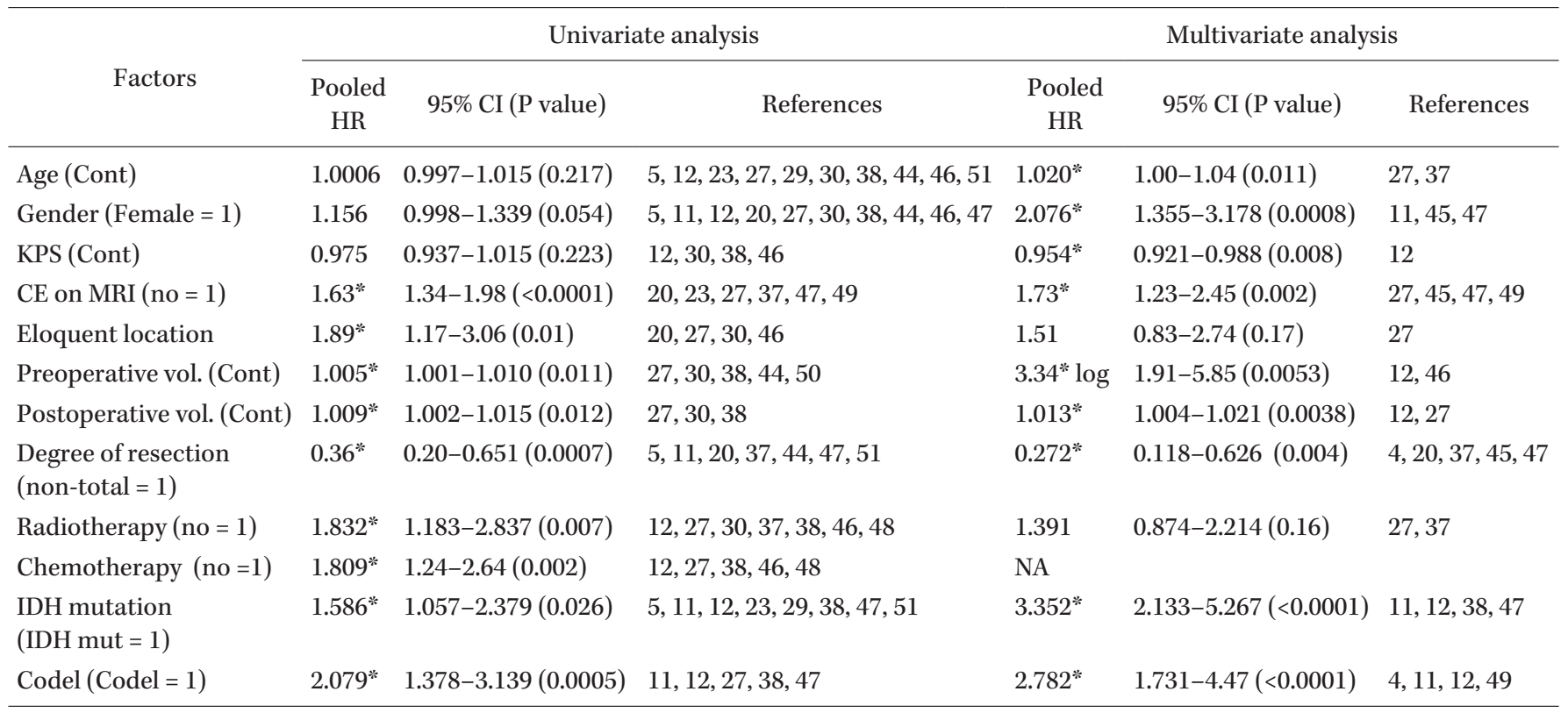

CE, contrast enhancement; Cont, continuous value; CI, confidence interval; HR, hazard ratio; KPS, Karnofsky performance status scale; log, logtransformed volume; NA, not available; Codel, 1p/19q codeletion; *, statistically significant.

high risk of bias when $\leq 5$ questions were answered "yes." Most of the studies had a low risk of bias. One study with a moderate risk that lacked some data of the total population was used only for percentage calculation and not for meta-regression analyses.

\section{Rate of malignant transformation}

We separately calculated the MaligR in Htrans and Ctrans. The pooled Ctrans rate was $34.6 \%$ (95\% CI: $27.9-$ 42.0, $I^{2}=94.4 \%$ ), whereas the pooled Htrans rate was $23.2 \%$ (95\% CI: 18.9-28.0, $I^{2}=89.8 \%$ ) (forest plots in Supplementary Figure 3). Although high heterogeneities $\left(I^{2}\right)$ were observed, no publication bias was detected by funnel plots (Supplementary Figure 3).

Meta-regression analyses showed the significant contribution of MFTime to the heterogeneity of Ctrans ( $\mathrm{P}=$ $0.008)$ and Htrans rates $(P=0.003)$ (Table 2). The regression lines in the Htras and Ctrans rates indicated an almost parallel increase against MFTime (Fig. 1A). The pooled MaligR in recurrent cases was $46.5 \%$ (95\% CI: 38.554.8) and $66.3 \%$ (95\% CI: 57.7-74.0) for Htrans and Ctrans, respectively.

Multivariate meta-regression was performed only for the Htrans rate, as there were too few studies and covariates for the Ctrans rate. OligR and AstroR in each study were significantly related to MaligR when the MFTime was incorporated (Table 1). The Htrans rate in diffuse astrocytomas was much higher than that in oligodendrogliomas (Fig. 1B). Other factors, including GTRate $(P=0.18)$, the radiotherapy rate $(\mathrm{P}=0.26)$, and the chemotherapy rate $(\mathrm{P}$ $=0.79)$, did not contribute to the heterogeneity in Htrans rates.

\section{Factors related to malignant transformation}

Pooled HRs in factors of malignant transformation were calculated without distinction of Htrans and Ctrans because they both increased in parallel with MFTime (Fig. 1 A).

We summarized the results in Table 3 (forest plots in Supplementary Figure 4). Crucial factors for malignant transformation were molecular markers (both IDHm and Codel), pre- and postoperative volume, and the extent of removal. These factors reached significance in pooled HRs in both univariate and multivariate analyses. Contrast enhancement on MRI also significantly affected malignant transformation. The age, gender, and KPS were possible factors, indicating a tendency in the pooled HRs of univariate analyses but only indicating significance in a few multivariate analyses. Tumors treated with adjuvant radiotherapy and chemotherapy exhibited an increased MaligR in the pooled results of univariate analyses but did not in that of multivariate analyses.

We investigated collinearity among GTRate, OligoR, AstroR, chemotherapy rate, and radiotherapy rate in each study. No significant correlations were found among them, except for between OligoR and AstroR $(\mathrm{P}<0.0005)$.

\section{MaligR in molecularly defined LGG}

Because the molecular subtypes are crucial in the transformation of LGG, we determined the rates in tumors of IDHm with and without Codel. The extracted data from Kaplan-Meier curves from four studies with Htrans ${ }^{423,26,27)}$ 
and four studies with Ctrans ${ }^{11,12,14,52)}$ are presented in Fig. 2 A-D. The synthesized curves from the extracted KaplanMeier curves indicated that the 10-year MPFS for IDHm without Codel was 30.4\% ( $\mathrm{n}=307$; 95\% CI: 22.2-39.0) with Htrans and $38.3 \%(\mathrm{n}=458$; 95\% CI: 32.3-44.3) with Ctrans, and that for IDHm with Codel was $71.7 \%(n=272 ; 95 \%$ CI: 61.7-79.5) with Htrans and 62.5\% ( $\mathrm{n}=400$; 95\% CI: 55.968.5) with Ctrans (Figs. 2E and 2F).

Two studies included only patients without adjuvant therapy after surgery: GTRate of $41.1 \%$ in the study by Cesselli et al. ${ }^{12)}$ and $52.7 \%$ in that by Jansen et al. ${ }^{3)}$ In other studies, patients underwent adjuvant radiotherapy or chemotherapy in $>40 \%$ of cases, with GTRate ranging from $10 \%$ to $70 \%$. Patients with Ctrans in Cesselli et al. ${ }^{12)}$ had a significantly shorter MPFS than cases in other studies regarding both IDHm non-Codel (Fig. 2A, post-hoc test, $\mathrm{P}=$ 0.017) and IDHm Codel cases (Fig. 2B, post-hoc test, $\mathrm{P}=$ 0.026).

We performed an analysis to determine the difference in the 10yMPFS by meta-regression. The study by Jaber et al. was excluded because of the short follow-up period. "No adjuvant therapy" was the only factor associated with a poor outcome in patients with Codel tumors (metaregression, $\mathrm{P}=0.003$ ), even combined with GTRate and diagnosis (Htrans or Ctrans) (multivariate meta-regression, P $<0.0005)$. There were no significant factors associated with tumors with IDHm without Codel.

\section{Discussion}

This is the first meta-analysis of malignant transformation in LGGs. We demonstrated that MaligR in both Htrans and Ctrans were increased almost linearly for $>10$ years after diagnosis. The rates were significantly affected by the pre- and postoperative tumor volumes, extent of resection, and both histological and molecular diagnoses. Diffuse astrocytomas and tumors with IDHm without Codel exhibited much higher MaligR than oligodendrogliomas or tumors with IDHm with Codel.

\section{The rate of malignant transformation}

Previous studies reported diverse MaligR and related factors during various follow-up periods. We first metaanalyzed these data. The major problem in the analyses was that there was no universal definition of malignant transformation. The majority of studies defined histologically proven progression as malignant changes, but recent studies often added clinical features, especially contrast enhancement on MRI, for the diagnosis. The former definition missed cases of malignant change that were not surgically treated at recurrence. However, the definition including new contrast enhancement on MRI sometimes overdiagnosed malignant transformation.8) Our study demonstrated that the rates obtained through the two methods increased almost in parallel, so we suspect that the true value lies between them, being approximately $40 \%$ at MFTime of 10 years (Fig. 1A).

The MaligR value was higher in astrocytomas than in oligodendrogliomas (Fig. 1B). However, the histological diagnosis system has been changing over the past 5 years, and molecular diagnoses have become more important. We determined 10-year MPFS in IDHm non-Codel tumor to be $30.4 \%$ with Htrans and $38.3 \%$ with Ctrans, and that in IDHm Codel to be $71.7 \%$ with Htrans and $62.5 \%$ with Ctrans. We did not examine MaligR in IDHw LGG as this tumor type might include heterogeneous specimens.

\section{Factors relating malignant transformation}

Patient age is an important prognostic factor for OS in LGG. ${ }^{9,12,27}$ This may be influenced by various confounding factors; for example, elderly patients may undergo less aggressive therapy or have serious comorbidities. Recent molecular studies have reported that IDHw astrocytomas, which are biologically more aggressive, arise more in older patients than IDHm astrocytomas. ${ }^{12)}$ Our study revealed a nonsignificant relationship between an older age and the incidence of MaligR in univariate analyses, but pooled results of two multivariate analyses indicated a significant relationship. ${ }^{27,37)}$ A similar result was observed in sex difference. In a multivariate analysis, only factors that showed significance or borderline significance in univariate analyses were included in the analyses. As a result, the pooled HR of multivariate analyses might have been skewed, as nonsignificant factors were not included. Both older age and male sex were possible factors associated with malignant transformation, but accumulation of further data is necessary.

Our study demonstrated that preoperative contrast enhancement on MRI was a strong predictor of early malignant progression in both univariate and multivariate analyses. Snyder et al. found that contrast enhancement on preoperative imaging was identified in $16 \%$ of LGGs. ${ }^{31)}$ They noted that the preoperative presence of a contrastenhancing tumor, which indicated no high-grade tumors by pathological examination, was predictive of MPFS ( $p<$ 0.0001 ). Narag et al. ${ }^{8)}$ reported that the postoperative appearance of new enhancement was associated with an inferior median OS, even in patients without malignant degeneration. Thus, even though new enhancement does not always indicate malignant changes, it may indicate that tumors are likely to be aggressive.

The pre- and postoperative volume and extent of resection were found to be significantly related to MaligR. As these three factors are related to one another, ${ }^{31,38)}$ it was difficult to determine which was the most crucial. However, these relationships would be modified if the molecular diagnoses were incorporated into the analyses. Patel et al. ${ }^{44)}$ reported that in an age-adjusted Cox regression model stratified by IDH mutation status, a greater extent of resection was only associated with prolonged MPFS in IDHw 
patients $(\mathrm{P}<0.001)$ but not in IDHm patients $(\mathrm{P}=0.83)$. The preoperative tumor volume had a statistically significant prognostic value for MPFS in the IDHm cohort $(\mathrm{P}=$ $0.01)$ but not in the IDHw cohort $(\mathrm{P}=0.23)$. Tom et al. ${ }^{11)}$ reported a significant relationship between MPFS and the degree of resection $(\mathrm{P}=0.002)$ and tumor size $(\mathrm{P}<0.001)$ in a univariate analysis. However, only the tumor size was significant in a multivariate analysis, combined with molecular classification and other factors. Thus, the initial tumor size appears to be a significant factor for MPFS, but further studies will be necessary to determine the role of resection of molecularly defined tumors.

The role of adjuvant radiotherapy or chemotherapy in malignant transformation was not distinct in the present study. Although pooled results from univariate analyses indicated a significant negative impact of both treatments, such findings were not demonstrated in multivariate analyses. This was likely due to selection bias, as patients with high-risk tumors were selected for adjuvant radiochemotherapy. However, the present study suggested that no adjuvant therapy was a factor associated with the transformation in Codel tumors (Fig. 2). Although the result was statistically significant, we had not initially planned to perform such an analysis. Because of the limited number of studies included, further investigation will be warranted.

Recent studies have warned that temozolomide treatment of LGG induces hypermutation, which may drive malignant progression. ${ }^{53,54)}$ Tom et al. ${ }^{11)}$ reported adjuvant temozolomide monotherapy as the only modifiable risk factor for malignant transformation among adult LGG cases. Aihara et al. $^{55}$ indicated that malignancies are rarely promoted by additionally acquired mutations or genomic aberrations at recurrence of oligodendrogliomas. Such molecular characteristics may account for the clinically benign nature of oligodendroglioma compared with other diffuse gliomas. Aoki et al. ${ }^{14)}$ proposed a mathematical model for malignant transformation of LGG. This model revealed that prompt adjuvant chemoradiotherapy prolonged MPFS in small IDHm LGGs, whereas the best treatment differed according to genetic alterations for large IDHm LGGs. These previous findings suggest that the natural history and complex treatment effects, either negatively or positively, influence the malignant transformation of LGG.

\section{Limitations}

Several limitations associated with the present study warrant mention. First, the accumulated data were obtained from retrospective studies with a moderate quality, which might have caused some bias. Second, we attempted to establish two definitions of malignant transformation. This might have been inappropriate in some cases, as the histological diagnosis rate for recurrences might differ among institutes, and some institutes employed various MRI sequences or positron emission tomography for the clinical diagnosis of malignant transformation. Furthermore, histological and radiological diagnosis of malignant transformation may be difficult after radiochemotherapy, whereas the diagnostic criteria may shift to molecular basis in future. However, the heterogeneity of the MaligR may be largely explained by MFTime and histological diagnosis. The rate of Htrans and Ctrans increased almost in parallel against MFTime. Third, the method of data extraction may be a limitation. Because of several data being missing, we calculated HRs using the method established by Tienarry et al. $^{13)}$ or obtained the values from Kaplan-Meier curves. The calculated data might have had a small range of error.

\section{Conclusion}

We evaluated MaligR in diffuse LGGs by a systematic review and meta-analysis. We established two definitions of malignant transformation: Htrans and Ctrans. The MaligRMFTime curves of Htrans and C trans were almost parallel. True MaligR was suspected to lie between the curves, at approximately $40 \%$ at the 10 -year follow-up. This rate was affected by the pre- and postoperative tumor volumes and extent of resection. An astrocytic histology and a molecular diagnosis of non-Codel were also strong factors affecting the transformation. The effect of adjuvant therapies could not be clarified.

\section{Supplementary Material}

https://doi.org/10.2176/jns-nmc.2021-0313

\section{Acknowledgments}

We would like to thank Mr. Brian Quinn (Japan Medical Communication) for editing a draft of this manuscript.

\section{Funding}

No specific grants were received for this research.

\section{Conflicts of Interest Disclosure}

The authors declare no conflicts of interest in association with the present study.

\section{References}

1) Louis DN, Ohagaki H, Wiestler OD, et al.: WHO classification of tumours of the central nervous system, International Agency for Research on Cancer, Lyon, 2016

2) Louis DN, Perry A, Wesseling P, et al.: The 2021 WHO Classification of Tumors of the Central Nervous System: A summary. Neuro Oncol 23: 1231-1251, 2021

3) Nakasu S, Notsu A, Nakasu Y: Prevalence of incidental meningiomas and gliomas on MRI: A meta-analysis and metaregression analysis. Acta Neurochir (Wien) 163: 3401-3415, 2021 
4) Jansen E, Hamisch C, Ruess D, et al.: Observation after surgery for low grade glioma: Long-term outcome in the light of the 2016 WHO classification. J Neurooncol 145: 501-507, 2019

5) Thon N, Eigenbrod S, Kreth S, et al.: IDH1 mutations in grade II astrocytomas are associated with unfavorable progression-free survival and prolonged postrecurrence survival. Cancer 118: 452460, 2012

6) Lima GL, Zanello M, Mandonnet E, Taillandier L, Pallud J, Duffau H: Incidental diffuse low-grade gliomas: From early detection to preventive neuro-oncological surgery. Neurosurg Rev 39: 377-384, 2016

7) Duffau H: Long-term outcomes after supratotal resection of diffuse low-grade gliomas: A consecutive series with 11-year followup. Acta Neurochir (Wien) 158: 51-58, 2016

8) Narang AK, Chaichana KL, Weingart JD, et al.: Progressive lowgrade glioma: Assessment of prognostic importance of histologic reassessment and MRI findings. World Neurosurg 99: 751-757, 2017

9) Capelle L, Fontaine D, Mandonnet E, et al.: Spontaneous and therapeutic prognostic factors in adult hemispheric World Health Organization Grade II gliomas: A series of 1097 cases: Clinical article. J Neurosurg 118: 1157-1168, 2013

10) Obara T, Blonski M, Brzenczek C, et al.: Adult diffuse low-grade gliomas: 35-year experience at the nancy france neurooncology unit. Front Oncol 10: 2020

11) Tom M, Park D, Wei W, et al.: Malignant transformation of adult low grade glioma: Risk factors and outcomes in the molecular era. Int J Radiat Oncol Biol Phys 105: S143, 2019

12) Cesselli D, Ius T, Isola M, et al.: Application of an artificial intelligence algorithm to prognostically stratify grade II gliomas. Cancers 12: 50, 2021

13) Tierney JF, Stewart LA, Ghersi D, Burdett S, Sydes MR: Practical methods for incorporating summary time-to-event data into meta-analysis. Trials 8: 16, 2007

14) Aoki K, Suzuki H, Yamamoto T, et al.: Mathematical modeling and mutational analysis reveal optimal therapy to prevent malignant transformation in grade II IDH-mutant gliomas. Cancer Res 81: 4861-4873, 2021

15) Ahmadi R, Dictus C, Hartmann C, et al.: Long-term outcome and survival of surgically treated supratentorial low-grade glioma in adult patients. Acta Neurochir (Wien) 151: 1359-1365, 2009

16) Bauman G, Fisher B, Watling C, Cairncross JG, Macdonald D: Adult supratentorial low-grade glioma: Long-term experience at a single institution. Int J Radiat Oncol Biol Phys 75: 1401-1407, 2009

17) Dahlrot RH, Kristensen BW, Hjelmborg J, Herrstedt J, Hansen S: A population-based study of low-grade gliomas and mutated isocitrate dehydrogenase 1 (IDH1). J Neurooncol 114: 309-317, 2013

18) El-Hateer H, Souhami L, Roberge D, et al.: Low-grade oligodendroglioma: an indolent but incurable disease? Clinical article. $J$ Neurosurg 111: 265-271, 2009

19) Etxaniz O, Carrato C, de Aguirre I, et al.: IDH mutation status trumps the Pignatti risk score as a prognostic marker in lowgrade gliomas. J Neurooncol 135: 273-284, 2017

20) Gousias K, Schramm J, Simon M: Extent of resection and survival in supratentorial infiltrative low-grade gliomas: Analysis of and adjustment for treatment bias. Acta Neurochir (Wien) 156: 327-337, 2014

21) Heo YJ, Park JE, Kim HS, et al.: Prognostic relevance of gemistocytic grade II astrocytoma: Gemistocytic component and MR imaging features compared to non-gemistocytic grade II astrocytoma. Eur Radiol 27: 3022-3032, 2017
22) Iwadate Y, Matsutani T, Hirono S, Ikegami S, Shinozaki N, Saeki $\mathrm{N}$ : IDH1 mutation is prognostic for diffuse astrocytoma but not low-grade oligodendrogliomas in patients not treated with early radiotherapy. J Neurooncol 124: 493-500, 2015

23) Jaber M, Ewelt C, Wölfer J, et al.: Is visible aminolevulinic acidinduced fluorescence an independent biomarker for prognosis in histologically confirmed (World Health Organization 2016) lowgrade gliomas? Neurosurgery 84: 1214-1224, 2019

24) Jung TY, Jung S, Moon JH, et al.: Early prognostic factors related to progression and malignant transformation of low-grade gliomas. Clin Neurol Neurosurg 113: 752-757, 2011

25) Juratli TA, Kirsch M, Robel K, et al.: IDH mutations as an early and consistent marker in low-grade astrocytomas WHO grade II and their consecutive secondary high-grade gliomas. J Neurooncol 108: 403-410, 2012

26) Karschnia P, Teske N, Dorostkar MM, et al.: Extent and prognostic value of MGMT promotor methylation in glioma WHO grade II. Sci Rep 10: 19758, 2020

27) Kavouridis VK, Boaro A, Dorr J, et al.: Contemporary assessment of extent of resection in molecularly defined categories of diffuse low-grade glioma: A volumetric analysis. J Neurosurg 133: 12911301, 2019

28) Koo T, Lim DH, Seol HJ, et al.: Impact of adjuvant treatments on survival in Korean patients with WHO grade II gliomas: KNOG 15-02 and KROG 16-04 intergroup study. J Neurooncol 140: 445455, 2018

29) Leu S, von Felten S, Frank S, Boulay JL, Mariani L: IDH mutation is associated with higher risk of malignant transformation in low-grade glioma. J Neurooncol 127: 363-372, 2016

30) Majchrzak K, Kaspera W, Bobek-Billewicz B, et al.: The assessment of prognostic factors in surgical treatment of low-grade gliomas: A prospective study. Clin Neurol Neurosurg 114: 11351144, 2012

31) Snyder LA, Wolf AB, Oppenlander ME, et al.: The impact of extent of resection on malignant transformation of pure oligodendrogliomas. J Neurosurg 120: 309-314, 2014

32) Suzuki A, Maruyama T, Nitta M, et al.: Evaluation of DNA ploidy with intraoperative flow cytometry may predict long-term survival of patients with supratentorial low-grade gliomas: Analysis of 102 cases. Clin Neurol Neurosurg 168: 46-53, 2018

33) Tanaka K, Sasayama T, Mizukawa K, et al.: Combined IDH1 mutation and MGMT methylation status on long-term survival of patients with cerebral low-grade glioma. Clin Neurol Neurosurg 138: 37-44, 2015

34) Turkoglu E, Gurer B, Sanli AM, et al.: Clinical outcome of surgically treated low-grade gliomas: A retrospective analysis of a single institute. Clin Neurol Neurosurg 115: 2508-2513, 2013

35) Youland RS, Brown PD, Giannini C, Parney IF, Uhm JH, Laack NN: Adult low-grade glioma: 19-year experience at a single institution. Am J Clin Oncol 36: 612-619, 2013

36) Zhang S, Chiang GC, Magge RS, et al.: Texture analysis on conventional MRI images accurately predicts early malignant transformation of low-grade gliomas. Eur Radiol 29: 2751-2759, 2019

37) Chaichana KL, McGirt MJ, Laterra J, Olivi A, Quiñones-Hinojosa A: Recurrence and malignant degeneration after resection of adult hemispheric low-grade gliomas. J Neurosurg 112: 10-17, 2010

38) Eseonu CI, Eguia F, ReFaey K, et al.: Comparative volumetric analysis of the extent of resection of molecularly and histologically distinct low grade gliomas and its role on survival. $J$ Neurooncol 134: 65-74, 2017

39) Fukuya Y, Ikuta S, Maruyama T, et al.: Tumor recurrence pat- 
terns after surgical resection of intracranial low-grade gliomas. $J$ Neurooncol 144: 519-528, 2019

40) Jakola AS, Myrmel KS, Kloster R, et al.: Comparison of a strategy favoring early surgical resection vs a strategy favoring watchful waiting in low-grade gliomas. JAMA 308: 1881-1888, 2012

41) Jungk C, Scherer M, Mock A, et al.: Prognostic value of the extent of resection in supratentorial WHO grade II astrocytomas stratified for IDH1 mutation status: A single-center volumetric analysis. J Neurooncol 129: 319-328, 2016

42) Ross DA, Tao S, Gultekin S, Ross AM: Lobar distribution of low grade oligodendroglioma: Distribution, molecular characteristics, and survival based upon location. J Cancer Ther 5: 1249, 2014

43) Jansen NL, Suchorska B, Wenter V, et al.: Dynamic 18F-FET PET in newly diagnosed astrocytic low-grade glioma identifies highrisk patients. J Nucl Med 55: 198-203, 2014

44) Patel T, Bander ED, Venn RA, et al.: The role of extent of resection in IDH1 wild-type or mutant low-grade gliomas. Neurosurgery 82: 808-814, 2018

45) Sakarunchai I, Sangthong R, Phuenpathom N, Phukaoloun M: Free survival time of recurrence and malignant transformation and associated factors in patients with supratentorial low-grade gliomas. J Med Assoc Thai 96: 1542-1549, 2013

46) Smith JS, Chang EF, Lamborn KR, et al.: Role of extent of resection in the long-term outcome of low-grade hemispheric gliomas. J Clin Oncol 26: 1338-1345, 2008

47) Gozé C, Blonski M, Le Maistre G, et al.: Imaging growth and isocitrate dehydrogenase 1 mutation are independent predictors for diffuse low-grade gliomas. Neuro Oncol 16: 1100-1109, 2014

48) Murphy ES, Leyrer CM, Parsons M, et al.: Risk factors for malignant transformation of low-grade glioma. Int J Radiat Oncol Biol
Phys 100: 965-971, 2018

49) Pallud J, Blonski M, Mandonnet E, et al.: Velocity of tumor spontaneous expansion predicts long-term outcomes for diffuse lowgrade gliomas. Neuro Oncol 15: 595-606, 2013

50) Weller J, Katzendobler S, Karschnia P, et al.: PCV chemotherapy alone for WHO grade 2 oligodendroglioma: Prolonged disease control with low risk of malignant progression. J Neurooncol 2021

51) Wen B, Fu F, Hu L, et al.: Subventricular zone predicts high velocity of tumor expansion and poor clinical outcome in patients with low grade astrocytoma. Clin Neurol Neurosurg 168: 12-17, 2018

52) Zeng L, Mei Q, Li H, Ke C, Yu J, Chen J: A survival analysis of surgically treated incidental low-grade glioma patients. Sci Rep 11: 8522, 2021

53) Johnson BE, Mazor T, Hong C, et al.: Mutational analysis reveals the origin and therapy-driven evolution of recurrent glioma. Science 343: 189-193, 2014

54) Yu Y, Villanueva-Meyer J, Grimmer MR, et al.: Temozolomideinduced hypermutation is associated with distant recurrence and reduced survival after high-grade transformation of low-grade IDH-mutant gliomas. Neuro Oncol 23: 1872-1884, 2021

55) Aihara K, Mukasa A, Nagae G, et al.: Genetic and epigenetic stability of oligodendrogliomas at recurrence. Acta Neuropathol Commun 5: 18, 2017

Corresponding author: Satoshi Nakasu, M.D.

Division of Neurosurgery, Omi Medical Center, 1660 Yabase-cho, Kusatsu, Shiga 525-8585, Japan.

e-mail: neuro-nakasu@kfx.biglobe.ne.jp 